\title{
The acoustic startle reflex as a tool for assessment of odor environment effects on affective states in laboratory mice
}

\author{
Hideaki INAGAKI and Takahiro USHIDA \\ Multidisciplinary Pain Center, Aichi Medical University, 1-1 Yazakokarimata, Nagakute, Aichi 480-1195, Japan
}

\begin{abstract}
Apart from self and conspecific odors, odors from other species also influence the affective states in laboratory mice (Mus musculus musculus) in their home cages and during experimental procedures, possibly inducing confusion and inconsistency in experimental data. Thus, it is important to detect the types of animal odors associated with housing, husbandry, and laboratory practice that can arouse different types of affective changes in mice. Here, we aimed to test the effectiveness of the acoustic startle reflex (ASR) in detecting changes in the affective states of laboratory mice due to animal-derived-odor as it has a non-zero baseline, and can be enhanced or attenuated by positive or negative affective shifts, respectively. We used ASR to examine the affective changes in mice that were induced by bedding odors and an alarm pheromone. The odor of bedding obtained from the mice' home cages significantly attenuated the ASR, suggesting positive affective shifts in the test mice, whereas that from bedding obtained from rat cages significantly enhanced the ASR, suggesting negative affective shifts. No significant changes in ASR were observed in mice presented with the odor of bedding obtained from cages of unfamiliar conspecifics. In contrast, there was significant ASR enhancement in mice exposed to volatile components of alarm pheromones trapped in water, suggesting negative affective shifts. Thus, our findings show that ASR may be a valuable tool in assessing the effects of odors on the affective states in laboratory mice.
\end{abstract}

Key words: alarm pheromone, bedding odor, predator odor, volatile substance

\section{Introduction}

Various routine husbandry and laboratory procedures can influence the physiology and behaviors of laboratory mice (Mus musculus musculus). For example, cage transport, individual housing, fasting, tail-tip sampling, and intraperitoneal injection significantly influence heart rate, blood pressure, body temperature, blood glucose level, and locomotor activity [1]. Furthermore, biochemical, hematological, and metabolic/endocrine parameters in mice are considerably influenced by cage density, diet, fasting duration, and venipuncture [2]. These facts suggest that the outcomes of physiological and/or behavioral experiments with laboratory mice are obviously affected by routine husbandry and laboratory procedures.

Odors are widely involved in a variety of major behaviors associated with the affective changes in mice such as territory marking, mating, food acquisition, alarm responses, and predation defenses [3-5]. Therefore, it has been considered that not only self and conspecific odors (e.g., bedding odor and alarm pheromone), but also odors from other species (e.g., odor from rats) influence affective states in laboratory mice, both in their home cages and during experimental procedures. This possibly induces confusion and inconsistency in experimental data obtained through physiological and/or behavioral test paradigms $[4,6,7]$. Thus, the detection of the different types of animal odors associated with housing, husbandry, and laboratory practice that can 
induce affective changes in mice should be an important aspect of experimental studies. However, quantitative and temporal regulation of sample presentation is comparatively difficult if conscious, free-moving mice are exposed to odors, particularly volatiles, in experiments to assess their affective states. Thus, there remain many issues to be addressed and understood about the effects of animal-derived odor on the affective states of laboratory mice.

The acoustic startle reflex (ASR) is a contraction of facial and skeletal muscles with eyelid closure, which is regulated by a simple reflexive neural pathway, in response to an abrupt and intense auditory stimulus, and has been demonstrated in humans and non-human mammals, including mice [8]. The ASR has a non-zero baseline and can be enhanced or attenuated by positive or negative affective shifts, respectively, in mice [8]. For example, enhanced ASR in mice was observed in the presence of an aversive conditioned cue generated by means of Pavlovian fear conditioning, termed "fearpotentiated startle" $[9,10]$. However, the ASR in mice was reduced by the presentation of a neutral stimulus cue associated with a reward in an appetitive-conditioning procedure, termed "pleasure-attenuated startle" [11]. In most ASR tests, startle amplitudes are recorded from mice placed inside a small animal holder wherein movement of the subject mouse is greatly limited [12]. Such experimental procedures for ASR tests are generally conducted in a relatively small soundproof test chamber, which can be easily closed and promptly ventilated [12]. Thus, we hypothesized that such experimental designs for ASR testing were suitable for data acquisition to assess affective changes in conscious, free-moving mice exposed to volatile stimuli derived from laboratory environment in a quantitatively and temporally regulated manner.

The aim of this study was to assess the effectiveness of the ASR test as a valuable tool to detect animal-derived volatiles that can possibly confuse experimental results by causing changes in the affective states of laboratory mice. We selected bedding odors and an alarm pheromone as common factors influencing the affective states in mice during routine laboratory protocols $[4,6$, 7] and examined the ASR changes induced by these stimuli.

\section{Materials and Methods}

\section{Experimental animals}

Fifty eight, 8-week-old, adult male mice (Slc:ddY; Japan SLC, Shizuoka, Japan) were used as subjects in this study. All animals were housed in pairs in wire- topped transparent cages $(320 \mathrm{~mm}$ length $\times 210 \mathrm{~mm}$ width $\times 130 \mathrm{~mm}$ height) with paper bedding. Mice were provided with water and food ad libitum and maintained on a $12 \mathrm{~h}$ light-dark cycle with the light extinguished at 1945 h. Cages were maintained at a constant temperature $\left(23 \pm 1^{\circ} \mathrm{C}\right)$ and humidity $(45-60 \%)$. This study was approved by the Animal Experiment Committee of Aichi Medical University. All animal care and experiments were performed in accordance with the committee's guidelines.

\section{Preparation and presentation of sample bedding}

Three kinds of paper bedding were prepared as test samples i.e. (1) Self bedding, obtained from home cages containing a male subject and its male pair partner; (2) Other bedding, obtained from other cages containing two 8-week-old male mice, neither of which were subjects of the ASR test; (3) Rat bedding, obtained from rat cages containing two 9-week-old male rats. All test sample beddings were collected $7 \mathrm{~d}$ after cage cleaning.

In each ASR test, a piece of sample paper bedding (10 $\mathrm{mm}$ length, $5 \mathrm{~mm}$ diameter) placed on a small disposable aluminum dish was presented to each subject (Self bedding, $n=8$; Other bedding, $n=8$; Rat bedding, $n=8$ ). In addition, a piece of clean, unused bedding was presented as a control sample bedding.

\section{Preparation and presentation of sample water}

As the mouse alarm pheromone is considered to be volatile and water soluble $[13,14]$, we trapped volatiles released from stressed mice in water droplets and used these as sample water, termed Stress water. Adult male mice (Slc:ddY, 9 weeks old, $n=6$ ) were used as pheromone donors, all of which were unfamiliar to subjects used in the experiments. Each donor was placed inside a polypropylene $50 \mathrm{ml}$ screw-cap conical tube (Eppendorf; Westbury, NY, USA) with 76 perforations (4 mm diameter). Subsequently, the conical tube containing the donor was set inside an acrylic box (100 mm length $\times 100$ $\mathrm{mm}$ width $\times 50 \mathrm{~mm}$ height; $2 \mathrm{~mm}$ thickness), the ceiling of which was sprayed with purified water $(1 \mathrm{ml})$. Immediately after that, 5 min tactile stimuli (each force of about $180 \mathrm{~g}$ ) with an interstimulus interval of 2 s (i.e., total 150 stimuli) were delivered to each donor's back using a stainless wire (500 mm length, $1 \mathrm{~mm}$ diameter) inserted through a hole ( $5 \mathrm{~mm}$ diameter) in the middle of the ceiling of the acrylic box and a perforated hole in the conical tube (Fig. 1), based on the same principle as that of the von Frey test [15]. The conical tube containing the donor mouse was removed 2 min after the last tactile stimulation. Then, the water droplets on the ceiling that contained the alarm pheromone were collected 


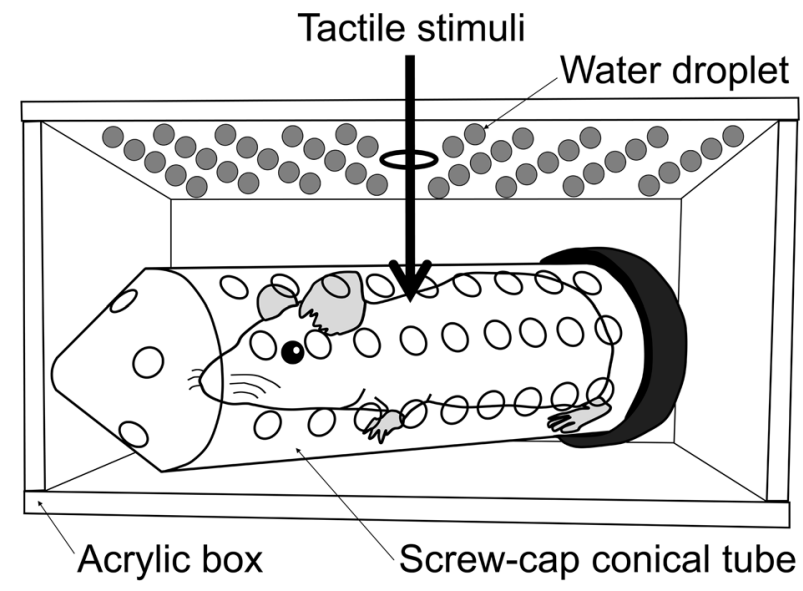

Fig. 1. A schematic diagram of the procedure for trapping volatile components of mouse alarm pheromone in purified water.

in a $2 \mathrm{ml}$ polypropylene tube (Eppendorf) using a glass bar and were stored at $-30^{\circ} \mathrm{C}$ until the next test day. Using the same procedure as that used for preparing Stress water, we prepared two additional types of sample water, termed Non-stress water and No animal water, using male mice (Slc:ddY, 9 weeks old, $n=5$ ) anesthetized with medetomidine hydrochloride (Domitol; Meiji Seika Pharma Co., Ltd., Tokyo, Japan; $0.3 \mathrm{mg} / \mathrm{kg}$, ip), midazolam (Dormicum; Astellas Pharma Inc., Tokyo, Japan; $4.0 \mathrm{mg} / \mathrm{kg}$, ip), and butorphanol (Vetorphale; Meiji Seika Pharma Co., Ltd.; $5.0 \mathrm{mg} / \mathrm{kg}$, ip) [16] for the former and no mice for the latter instead of stressed male mice.

In each ASR test, sample water $(0.45 \mathrm{ml})$ was dropped onto three sheets of clean, unused filter paper $(30 \mathrm{~mm}$ length $\times 20 \mathrm{~mm}$ width, laid one on top of another) on a disposable aluminum dish and presented to each subject (Stress water, $n=12$; Non-stress water, $n=10$; No animal water, $n=12$ ). Purified water was used as control sample water.

\section{The ASR test}

The startle apparatus and software (StartleReflexSystem 2004; O’Hara \& Co., Tokyo, Japan) were used in this study. The subjects were 58 adult, 8 -week-old male mice. The experiment lasted for five consecutive days. On days 1 and 2, each subject was handled for $5 \mathrm{~min}$ in the experimental room (temperature $23 \pm 1^{\circ} \mathrm{C}$, humidity 45-60\%). Then, the subject was acclimatized to the animal holder and the experimental apparatus. The holder consisted of a plastic inner cylinder $(110 \mathrm{~mm}$ length, $30 \mathrm{~mm}$ outside diameter, $28 \mathrm{~mm}$ inside diameter, $1 \mathrm{~mm}$ thickness), an acrylic outer cylinder (200 mm length, $50 \mathrm{~mm}$ outside diameter, $46 \mathrm{~mm}$ inside diameter, $2 \mathrm{~mm}$ thickness), and an acrylic bottom plate $(230 \mathrm{~mm}$ length $\times 120 \mathrm{~mm}$ width; $2 \mathrm{~mm}$ wall thickness) to support the outer cylinder. We inserted each subject into the inner cylinder of the animal holder head first, which was fixed inside the outer cylinder. Immediately after that, the animal holder containing the subject was attached to a platform in a dark, soundproof test chamber $(480 \mathrm{~mm}$ length, $350 \mathrm{~mm}$ width, $370 \mathrm{~mm}$ height) with background noise ( $65 \mathrm{~dB}$ white noise), which was produced by a speaker located at the rear of the test chamber ceiling, and maintained there for $10 \mathrm{~min}$. On day 3 , subjects were handled for $5 \mathrm{~min}$, followed by acclimatization to the entire ASR test procedure in the experimental room. Each mouse was placed inside the same individual animal holder as that used on days 1 and 2. Then, the animal holder containing each subject was attached to the platform in the dark soundproof test chamber with background noise, as on days 1 and 2. Following this, the ASR test, consisting of a baseline trial and a test trial, was initiated. During the baseline trial, after an initial $300 \mathrm{~s}$ acclimation period, each subject was exposed to 50 auditory stimuli ( $120 \mathrm{~dB}, 40 \mathrm{~ms}$ white noise) with a randomized interstimulus interval of $10-20 \mathrm{~s}$. The auditory stimuli were delivered through a speaker on the ceiling of the test chamber, located $140 \mathrm{~mm}$ above the top of the animal holder. All auditory stimuli were made through an interface (WP-1020; O'Hara \& Co.) under the control of the software on a personal computer. Subsequently, we set a control sample across the perforated front of the inner cylinder (eight perforations, each of 2 $\mathrm{mm}$ diameter) of the animal holder at a distance of about $25 \mathrm{~mm}$ below the nose of the mouse. After that, the test trial was conducted in the same manner as the baseline trial. The sample was left at a distance of about $25 \mathrm{~mm}$ below the nose of the mouse during the test trial. On days 4 and 5, the subjects underwent the same ASR test, which consisted of the presentation of control samples or test samples during the test trials, in a counterbalanced order. During the baseline and test trials, ASR data were recorded. In short, the subject's movements within the holder resulted in displacements of an accelerometer affixed to the platform, and the voltage output of the accelerometer was digitized and recorded via the personal computer software. The startle amplitude was defined as the maximal peak-to-peak voltage that occurred during the first $0.2 \mathrm{~s}$ after the onset of the startleeliciting auditory stimulus. All experimental procedures were conducted between 1300 and $1700 \mathrm{~h}$.

\section{Data analysis}

For data analyses, we defined individual baseline and test data as the mean amplitude of the last 40 responses in each trial, because we considered the habituation of 
each subject to the auditory stimuli and eliminated the first 10 responses. The percentage increase in amplitude between the test data $(T)$ and baseline data $(B)$ was calculated as $(T / B-1) \times 100$ for each subject. All data were displayed as the mean \pm SE. Data on days 4 and 5 were statistically compared within each experimental group using Wilcoxon signed-rank test (EZR statistical software [17]). The criterion for statistical significance was set at $P<0.05$ for all comparisons.

\section{Results}

The presentation of Self bedding to mice $(n=8)$ induced a significant percentage decrease in ASR values as compared to those presented with control bedding $(P<0.05$; Fig. 2A), whereas no significant difference was observed in the mice $(n=8)$ presented with Other bedding (Fig. 2B). The presentation of Rat bedding to mice $(n=8)$ induced a significant percentage increase in ASR values as compared to those presented with control bedding $(P<0.05$; Fig. 2C)

When Stress water was presented to mice $(n=12)$, there
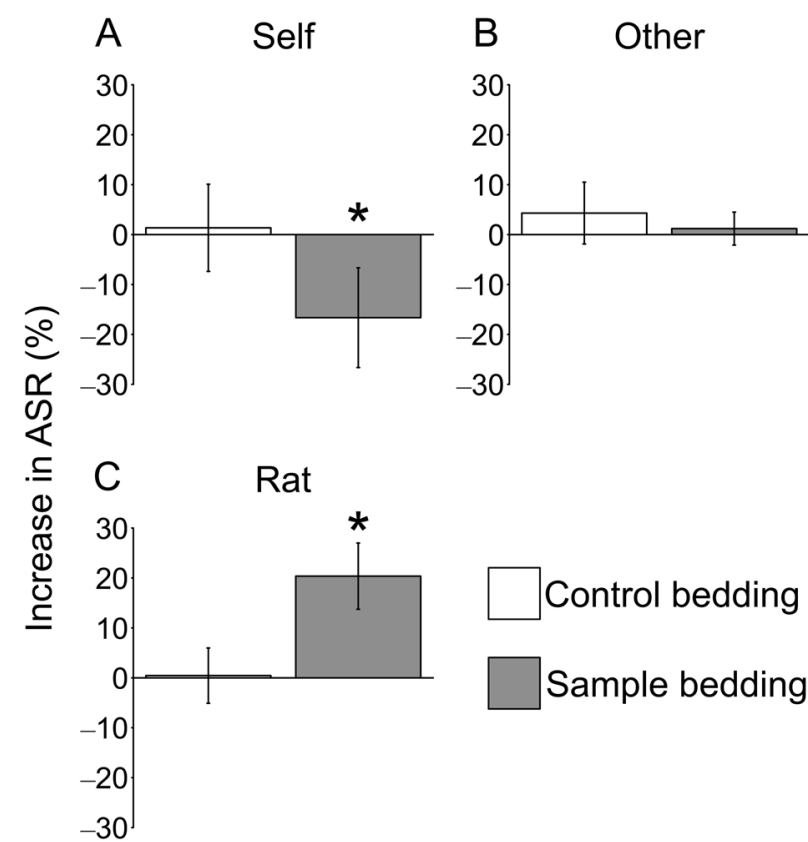

Fig. 2. The percentage increase in amplitude between the baseline data and test data for acoustic startle reflex (ASR). Subject mice were presented with sample bedding or control bedding (clean and unused bedding) in counterbalanced order between the baseline trial and the test trial during two consecutive experimental days. Samples presented were as follows: Self bedding, obtained from home cages containing a subject and its male pair partner (A Self, $n=8$ ); Other bedding, obtained from other cages containing two male mice, both of whom were not subjects of the ASR test (B Other, $n=8$ ); Rat bedding, obtained from rat cages containing two male rats ( $\mathrm{C}$ Rat, $\mathrm{n}=8$ ). Each bar represents the mean $\pm \mathrm{SE} ; * P<0.05$ versus control (Wilcoxon signed-rank test). was a significant percentage increase in ASR values compared to those presented with control water $(P<0.05$; Fig. 3A); however, no significant differences were observed when both Non-stress water $(n=10)$ and No animal water $(n=12)$ were presented to the subjects (Figs. $3 \mathrm{~B}$ and $\mathrm{C}$ ).

\section{Discussion}

In the present study, we assessed ASR changes in mice induced by bedding odors and an alarm pheromone, both of which are typical animal-derived odor stimuli associated with routine laboratory protocols. After presentation with three kinds of bedding odors, three different ASR test results were obtained. The odor of bedding obtained from the subjects' home cages significantly attenuated the ASR, suggesting positive affective shifts in the subject mice. In contrast, the odor of bedding obtained from rat cages significantly enhanced the ASR, suggesting negative affective shifts in the subject mice. No significant changes in ASR were observed in mice presented with the odor of bedding obtained from cages of unfa-

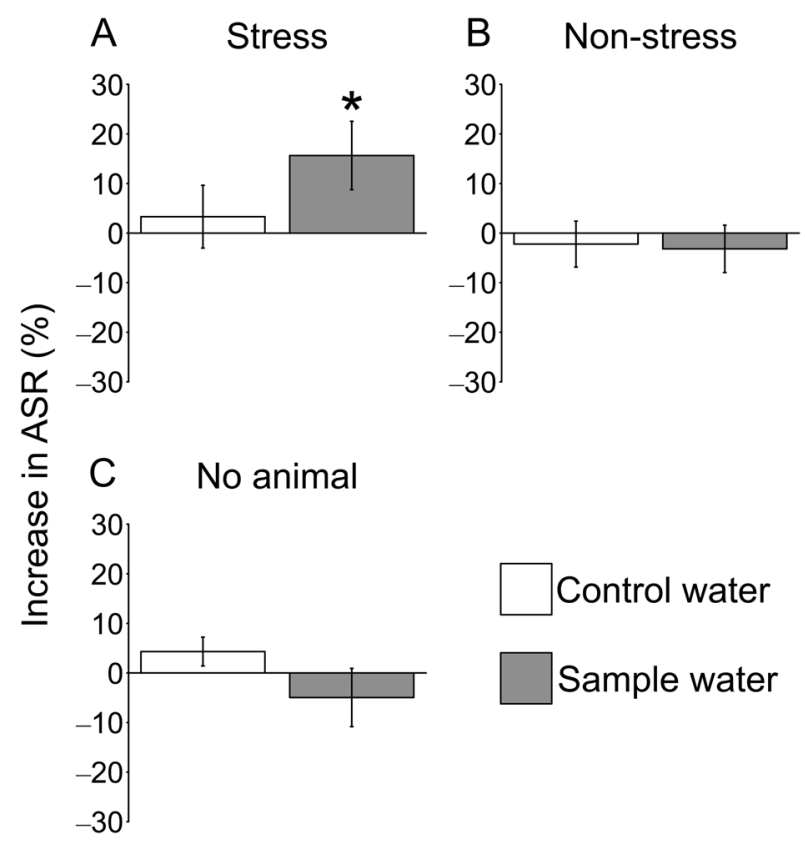

Fig. 3. The percentage increase in amplitude between the baseline data and test data for acoustic startle reflex (ASR). Subject mice were presented with sample water or control water (purified water) in counterbalanced order between the baseline trial and the test trial during two consecutive experimental days. Samples presented were as follows: Stress water, trapping volatiles released from stressed mice (A Stress, $n=12$ ); Non-stress water, trapping volatiles released from anesthetized mice ( $B$ Non-stress, $n=10$ ); No animal water, trapping volatiles in air without animals $(\mathrm{C} N o$ animal, $\mathrm{n}=12$ ). Each bar represents the mean $\pm \mathrm{SE} ; * P<0.05$ versus control (Wilcoxon signed-rank test). 
miliar conspecifics. However, there was significant ASR enhancement in subject mice exposed to volatile components of the alarm pheromone trapped in water, suggesting negative affective shifts in mice.

The present data demonstrate that the ASR test may be an effective paradigm to investigate the kinds of volatiles from beddings that can influence affective states in mice. In this study, the attenuation of ASR in mice, suggesting a positive affective shift, was observed when subjects were presented with volatiles from beddings of their home cages. This phenomenon may be explained as "social buffering", or a reduction of stress responses in animals induced by signals from social conspecific partners [18]. In the case of mice, olfaction is one of the important senses in relation to social buffering [19], wherein only volatiles obtained from familiar animals can induce reduction of stress responses [20]. These facts correspond to the present findings that unfamiliar volatiles derived from beddings in other cages could not change ASR, but familiar ones from home cages with one cage mate decreased it. In contrast, the enhancement of ASR in mice, suggesting negative affective shift, was observed when subjects were presented with volatiles from beddings in rat cages. This finding reflects the fact that one of the well-known predators of mice is the rat [3], which can induce anxiety-related defensive responses in mice, even when subjects were exposed to rats indirectly (e.g., across wire mesh barrier) in experimental conditions [21-23]. This experimental design indicates that olfaction is probably a main sensory modality related to negative affective shifts in mice on the basis of detection of volatiles released from rats, although possible contributions of other senses such as vision and hearing are also considered. Taken together, it is strongly suggested that the ASR test is a reliable tool to assess the effects of bedding-derived volatiles on affective states in mice during routine laboratory protocols that can possibly confuse the results of experiments that use laboratory mice.

This study also demonstrated that the ASR test may be effective in investigating the anxiogenic effects of the alarm pheromone in mice. The alarm pheromone is known to be released from mice in stressful conditions and to induce anxiety-related behaviors and high corticosterone levels in receiver mice $[13,14]$. It is shown that neurons of the Grueneberg ganglion, an olfactory subsystem located at the tip of the nose, detect the main component of this pheromone that is volatile and watersoluble $[13,14,24]$. The corresponding results regarding such biological and chemical characteristics were also shown in this study, namely, volatiles released from stressed mice were trapped in water droplets without direct contact and could induce significant ASR enhancement in receivers presented with sample water at a distance of a few centimeters from the nose. In reference to chemical alarm communication in laboratory animals, the ASR test is moreover indicated to be an effective paradigm to investigate anxiogenic effects of the alarm pheromone in rats. The rat alarm pheromone is released from the perianal region of donor rats and is trapped in water droplets without direct contact [25]. In line with these facts, two kinds of aldehydes, volatile and watersoluble, have been identified as the main pheromone components that are presumably perceived simultaneously by recipient rats via the main olfactory and vomeronasal systems, respectively [26]. The rat alarm pheromone can induce ASR enhancement in receiver rats presented with pheromone-containing sample water at a distance from the nose in the same manner as the mouse alarm pheromone in this study [27]. Thus, there is a possibility that the ASR test is a very useful paradigm to assess negative affective changes in laboratory animals that are caused by alarm pheromones of conspecifics that are handled, moved, or restrained during ordinary laboratory protocols and may confuse experimental results.

The changes in the ASR observed in this study can be attributed to the neuromechanism related to affective shifts via olfaction. The attenuation of ASR amplitude could be explained by the social-buffering effect of home cage odor. Moreover, it was considered that the enhancement in ASR amplitude was caused by predator odor and alarm pheromone. The odor-induced social buffering in rodents is suggested to be mainly mediated by the neural root from the main olfactory system to the amygdala in the limbic system [18], and this is also suggested to have an important role in affective shifts in mice evoked by predator odor [28]. The vomeronasal system and limbic system including the amygdala are considered the main contributors to the biological effects of rat alarm pheromone $[26,29]$. The amygdala is also a functionally significant brain region in terms of changes in the ASR amplitude reflecting the affective states (e.g., fear) in rats [30]. Overall, it is presumable that the amygdala is one of the most important brain regions mainly involved in the modulation of changes in the ASR observed in this study. However, further studies are necessary to clarify this issue.

It has been reported that alarm pheromones could be produced by laboratory mice under various types of stress conditions. Several studies have revealed the release of volatile alarm substances from mice that received substantial aversive stimuli; for example, intraperitoneal injection of hypertonic saline, electric foot shook, $\mathrm{CO}_{2}$ euthanasia, confinement stress, and cold 
stress $[13,14,31,32]$. In the present study, pheromone donor mice were confined and received tactile stimuli, also considered as considerable stress. However, it is unclear if more mild stresses such as low-intensity handling can elicit the release of alarm pheromone in mice. It is necessary to address this issue in future studies, as husbandry and laboratory protocols routinely include such mild handling of mice, which should be performed more carefully to prevent presumable confusion of physiological and/or behavioral experimental data, given that alarm pheromone release could be induced by such routine procedures.

In conclusion, we showed that the ASR test might be effective in assessing affective changes in laboratory mice exposed to volatile components of odors from cage beddings and the alarm pheromone. In ASR tests, presentation of volatile samples seems to be easy to perform in a quantitatively and temporally regulated manner. Therefore, it is suggested that the ASR test can become one of the valuable tools for detecting animal-derived odors that confuse experimental results and reduce the validity of experimental data by causing unknown effects on the affective states of mice during routine laboratory protocols. This would result in a reduction in the number of animals used and an improvement in laboratory animal welfare. Nevertheless, our present study has limitations and a number of questions remain to be answered; for example, clarifying the effect of not only human odor during husbandry procedures or experiments but also non-animal odor, such as disinfectants, on the affective states in laboratory mice.

\section{Acknowledgments}

This work was supported by JSPS KAKENHI Grant Number 16K07088.

\section{References}

1. Gerdin AK, Igosheva N, Roberson LA, Ismail O, Karp N, Sanderson M, et al. Experimental and husbandry procedures as potential modifiers of the results of phenotyping tests. Physiol Behav. 2012; 106: 602-611. [Medline] [CrossRef]

2. Champy MF, Selloum M, Piard L, Zeitler V, Caradec C, Chambon $\mathrm{P}$, et al. Mouse functional genomics requires standardization of mouse handling and housing conditions. Mamm Genome. 2004; 15: 768-783. [Medline] [CrossRef]

3. Latham N, Mason G. From house mouse to mouse house: the behavioural biology of free-living Mus musculus and its implications in the laboratory. Appl Anim Behav Sci. 2004; 86: 261-289. [CrossRef]

4. Olsson IAS, Nevison CM, Patterson-Kane EG, Sherwin CM, Van De Weerd HA, Würbel H. Understanding behaviour: the relevance of ethological approaches in laboratory animal science. Appl Anim Behav Sci. 2003; 81: 245-264. [CrossRef]

5. Arshamian A, Laska M, Gordon AR, Norberg M, Lahger C,
Porada DK, et al. A mammalian blood odor component serves as an approach-avoidance cue across phylum border - from flies to humans. Sci Rep. 2017; 7: 13635. [Medline] [CrossRef]

6. López-Salesansky N, Mazlan NH, Whitfield LE, Wells DJ, Burn CC. Olfaction variation in mouse husbandry and its implications for refinement and standardization: UK survey of animal scents. Lab Anim. 2016; 50: 362-369. [Medline] [CrossRef]

7. Bind RH, Minney SM, Rosenfeld S, Hallock RM. The role of pheromonal responses in rodent behavior: future directions for the development of laboratory protocols. J Am Assoc Lab Anim Sci. 2013; 52: 124-129. [Medline]

8. Koch M. The neurobiology of startle. Prog Neurobiol. 1999; 59: 107-128. [Medline] [CrossRef]

9. Jones SV, Heldt SA, Davis M, Ressler KJ. Olfactory-mediated fear conditioning in mice: simultaneous measurements of fear-potentiated startle and freezing. Behav Neurosci. 2005; 119: 329-335. [Medline] [CrossRef]

10. Daldrup T, Remmes J, Lesting J, Gaburro S, Fendt M, Meuth $\mathrm{P}$, et al. Expression of freezing and fear-potentiated startle during sustained fear in mice. Genes Brain Behav. 2015; 14: 281-291. [Medline] [CrossRef]

11. Friemel CM, Zimmer A, Schneider M. The CB1 receptor as an important mediator of hedonic reward processing. Neuropsychopharmacology. 2014; 39: 2387-2396. [Medline] [CrossRef]

12. Ioannidou C, Marsicano G, Busquets-Garcia A. Assessing prepulse inhibition of startle in mice. Bio Protoc. 2018; 8: e2789. [CrossRef]

13. Brechbühl J, Moine F, Klaey M, Nenniger-Tosato M, Hurni $\mathrm{N}$, Sporkert F, et al. Mouse alarm pheromone shares structural similarity with predator scents. Proc Natl Acad Sci USA. 2013; 110: 4762-4767. [Medline] [CrossRef]

14. Brechbühl J, Klaey M, Broillet MC. Grueneberg ganglion cells mediate alarm pheromone detection in mice. Science. 2008; 321: 1092-1095. [Medline] [CrossRef]

15. Deuis JR, Dvorakova LS, Vetter I. Methods used to evaluate pain behaviors in rodents. Front Mol Neurosci. 2017; 10: 284. [Medline] [CrossRef]

16. Kawai S, Takagi Y, Kaneko S, Kurosawa T. Effect of three types of mixed anesthetic agents alternate to ketamine in mice. Exp Anim. 2011; 60: 481-487. [Medline] [CrossRef]

17. Kanda Y. Investigation of the freely available easy-to-use software 'EZR' for medical statistics. Bone Marrow Transplant. 2013; 48: 452-458. [Medline] [CrossRef]

18. Kiyokawa Y, Hennessy MB. Comparative studies of social buffering: a consideration of approaches, terminology, and pitfalls. Neurosci Biobehav Rev. 2018; 86: 131-141. [Medline] [CrossRef]

19. Klein B, Bautze V, Maier AM, Deussing J, Breer H, Strotmann J. Activation of the mouse odorant receptor 37 subsystem coincides with a reduction of novel environment-induced activity within the paraventricular nucleus of the hypothalamus. Eur J Neurosci. 2015; 41: 793-801. [Medline] [CrossRef]

20. Liu H, Yuan TF. Physical interaction is required in social buffering induced by a familiar conspecific. Sci Rep. 2016; 6: 39788. [Medline] [CrossRef]

21. Zhou P, Xu HS, Li MM, Chen XD, Wang J, Zhou HB, et al. Mechanism of nitric oxide and acid-sensing ion channel 1a modulation of panic-like behaviour in the dorsal periaqueductal grey of the mouse. Behav Brain Res. 2018; 353: 32-39. [Medline] [CrossRef]

22. Pentkowski NS, Tovote P, Zavala AR, Litvin Y, Blanchard $\mathrm{DC}$, Spiess J, et al. Cortagine infused into the medial prefrontal cortex attenuates predator-induced defensive behaviors and Fos protein production in selective nuclei of the amygdala in male CD1 mice. Horm Behav. 2013; 64: 519-526. [Medline] [CrossRef]

23. Calvo-Torrent A, Brain PF, Martinez M. Effect of predatory 
stress on sucrose intake and behavior on the plus-maze in male mice. Physiol Behav. 1999; 67: 189-196. [Medline] [CrossRef]

24. Moine F, Brechbühl J, Nenniger Tosato M, Beaumann M, Broillet MC. Alarm pheromone and kairomone detection via bitter taste receptors in the mouse Grueneberg ganglion. BMC Biol. 2018; 16: 12. [Medline] [CrossRef]

25. Kiyokawa Y, Kikusui T, Takeuchi Y, Mori Y. Alarm pheromone that aggravates stress-induced hyperthermia is soluble in water. Chem Senses. 2005; 30: 513-519. [Medline] [CrossRef]

26. Inagaki H, Kiyokawa Y, Tamogami S, Watanabe H, Takeuchi Y, Mori Y. Identification of a pheromone that increases anxiety in rats. Proc Natl Acad Sci USA. 2014; 111: 18751-18756. [Medline] [CrossRef]

27. Inagaki H, Nakamura K, Kiyokawa Y, Kikusui T, Takeuchi Y, Mori Y. The volatility of an alarm pheromone in male rats. Physiol Behav. 2009; 96: 749-752. [Medline] [CrossRef]
28. Kondoh K, Lu Z, Ye X, Olson DP, Lowell BB, Buck LB. A specific area of olfactory cortex involved in stress hormone responses to predator odours. Nature. 2016; 532: 103-106. [Medline] [CrossRef]

29. Kiyokawa Y, Kodama Y, Kubota T, Takeuchi Y, Mori Y. Alarm pheromone is detected by the vomeronasal organ in male rats. Chem Senses. 2013; 38: 661-668. [Medline] [CrossRef]

30. Davis M, Walker DL, Miles L, Grillon C. Phasic vs sustained fear in rats and humans: role of the extended amygdala in fear vs anxiety. Neuropsychopharmacology. 2010; 35: 105-135. [Medline] [CrossRef]

31. Rottman SJ, Snowdon CT. Demonstration and analysis of an alarm pheromone in mice. J Comp Physiol Psychol. 1972; 81: 483-490. [Medline] [CrossRef]

32. Moynihan JA, Karp JD, Cohen N, Ader R. Immune deviation following stress odor exposure: role of endogenous opioids. $\mathrm{J}$ Neuroimmunol. 2000; 102: 145-153. [Medline] [CrossRef] 